\title{
Fault Location on Transmission line using Wavelet Transform and Artificial Neural Network
}

\author{
Amit M. Paikrao ${ }^{1}$, Abhijit S. Pande ${ }^{2}$ \\ Department of Electrical Engineering,, P. E. S. College of Engineering, Aurangabad, M.S, INDIA
}

\begin{abstract}
Finding and designing new methods for determining type and exact location of faults in power system has been a major subject for power system protection. One of the main capabilities that can improve the efficiency of new protection relays in power systems is fault location. In this paper wavelet transform along with neural networks is used for determiningfault location in Transmission lines. In the present work, theauthors have developed an algorithm for locating eleven types offaults over a 100\% of line length. The extraction of features of voltage signals and current signals by wavelet transform and subjecting it to artificial neural network, the fault location is calculated. Thealgorithm has been developed keeping in view the pragmaticshurdle of the multiple estimation which have been successfullytackled.
\end{abstract}

Keywords: Fault Location, Neural Networks, Transmission Line, Wavelet Transform

\section{Introduction}

Now a days power supply has become the business commodity, to remain in competition, the uninterrupted power supply must be given to the consumers. Power supply network consists of generation, transmission and distribution section, faults on any of these may lead to interruption of supply to consumer. The main function of the electrical transmission and distribution systems is to transport electrical energy from the generation unit to the customers[5]. Generally, when fault occurs on transmission lines, detecting fault is necessary for power system in order to clear fault before it increases the damage to the power system. The demand for reliable service has led to the development of technique of locating faults. During the course of recent years, the development of the fault diagnosis has been progressed with the applications of signal processing techniques and results in transient based techniques. It has been found that the wavelet transform is capable of investigating the transient signals generated in power system[1].

In recent years, there have been many activities in usingfault generated travelling wave methods for fault location andprotection. The travelling wave current-based fault locationscheme in which the distance to fault is determined by thetime differences measured at the sending end between anincident wave and the corresponding wave reflected from thefault have been developed for permanent faults in transmission networks[1][2][6]. However, due to the limitation of the bandwidth of theconventional CT (up to a few $\mathrm{GHz}$ ) and VT (up to $50 \mathrm{kHz}$ ), the accuracy of fault location provided by such a scheme is notsatisfactory for a power cable. Also there have been many activities in using power frequency (low frequency) for faultlocation and protection. However, in such techniques which arebased on power frequency signals, some useful informationassociated with high frequencies in transient condition ismissed. In association with wavelet transform the artificial intelligence can be used in locating faults on power cable by means of neural networks [3]. Although this method is complex yet speed for fault location is increased.

\section{Development Of Power System Model}

The work presented in this paper deals with fault distancelocation using WT and artificial neural network (ANN) for 11 types of faultsi.e. L-G faults, L-L-G faults, L-L faults, L-L-L faults, and L-L-L-Gfault in transmission lines.The study involve a $132 \mathrm{KV}$ transmission line of "Kagajipura-Kannad, Aurangabad (M.S.)" in India, $43 \mathrm{~km}$ length as a studied system. The work reports the results ofextensive "offline" studies using the Simulink/MATLAB and its associated toolboxes: Simulink, SimPowerSystems and Neural Network Toolbox [neural tool]. This protection scheme has been developed for transmission line using fundamental components of three-phase voltages and currents. Power system model is simulated as shown in Fig. 1. 


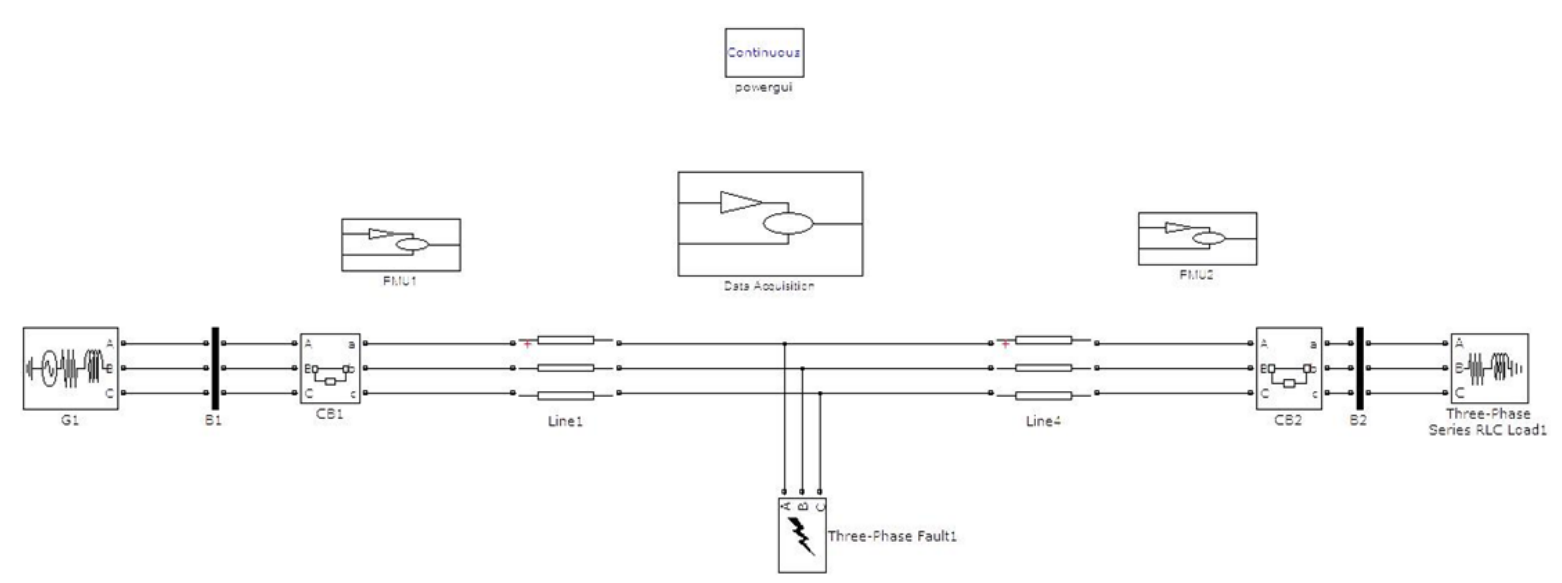

Fig.1 simulation of proposed model

In proposed model, Segments of distributed parameters are connected together to form the $100 \%$ of the line length i.e. $43 \mathrm{Km}$. Voltage of $132 \mathrm{KV}$ is generated by generating source which is given to proposed line. On the sending end side 3-phase circuit breaker is connected to control the switching phenomenon. At this end current and voltage with and without fault are measured with the help of PMU. On other hand receiving end has load $(20.8 \mathrm{e} 3 \mathrm{Kw}, 1300 \mathrm{KVAr})$ through 3-phase circuit breaker. The relaying function ofline is done with help of Phase Modulation Units (PMU) 1 and PMU 2[3] for each bus i.e. at B1 and B2. The data sensed by PMU1 and PMU2 is given to the data Acquisition block. The line parameters are as per appendix-1. The Wavelet transform decomposes voltage and current in 6 levels, and neural network identified the fault and location of fault.

\section{Feature Extraction Using Wavelet Transform}

Algorithms made for feature extraction are valuable tools, which transform high dimensional data to a lower one with anequivalent information content. It is used to reduce thedimensionality of data, thereby reducing the complexity ofclassification or regression scheme. Particularly wavelet transformsplits a given signal into a detail andan approximation by passing it through high pass and low passfilters. The approximations are obtained from first level splitsinto new detail and approximations and this process is repeateduntil required level of decomposition is achieved [4], in simple words if a data provided is of $1000 \mathrm{~Hz}$, after passing it through high pass and low pass filters, it will get spliced into $0-500 \mathrm{~Hz}$ of low band frequency and $500-1000 \mathrm{~Hz}$ as a high band frequency by Low Pass Filters, and High pass Filters respectively. Again low band pass is sliced into two parts of $0-250 \mathrm{~Hz}$, and $250-500 \mathrm{~Hz}$ and so on. Means, for whole $1000 \mathrm{~Hz}$ data we have $0-250,250-500,500-$ $1000 \mathrm{~Hz}$. In the present work, theauthors have utilized the six-level decomposition of thevoltage signals and current signals.Here, db1 (doubechies1)[ten lect.]as mother wavelet is selected as mother wavelet. This decomposition of signals helped to build algorithm for wavelet transform [1][4] which presents more possibilities forsignal processing. The features extracted by this are feed into neural network.

\section{Fault Location Based On Ann}

ANN have emerged as a powerful pattern recognition technique and act on data by detecting some form of underlying organisation not explicitly given or even known by human experts and it possesses certain features which are not attainable by the conventional methods. A single artificial neural network for fault distance location (FDL) of all the eleven types of faults under varying power system operating conditions has beendeveloped.

The implementation procedures for designing the neuralnetwork for fault distance location estimation are as follows [1][2].

Step 1: Obtain input data and target data from the simulation.

Step 2: Assemble and pre-process the training data for singleand modular ANN-based FDL.

Step 3: Create the network architecture and trainthe networkuntil conditions of network setting parameters are reached.

Step 4: Test and performance analysis.

Step 5: Stored the trained network.

Steps 1-5 are offlineprocesses. Next, the network is ready to test with the newinput. MATLAB is used to normalize the input signals. For trainingpattern or input matrix formation, the post-fault samples (elevenin number) of fundamental components of three-phasevoltages and currents are extracted. Using Simulink and SimPowerSystem toolbox of MATLAB all the eleven types of faults at different fault locationsbetween 0 and $100 \%$ of line length have been simulated, the total number of faults simulated is 583at different location and 
different faults of which $80 \%$ of simulation are given to form the training data set for neural network, and remaining for testing data.

In this paper supervised, multi-layer feed-forward networks, as, a three-layer network (input, one hidden and output layers) is used and Levenberg-Marquardt (LM) learning algorithm is used in the complete fault classification and fault location for the network. Training matrices were built in such a way that thenetwork trained produces an output corresponding to thefault distance location.The proposed methodology of faultdistance location using ANN is depicted in Figure 2.

\section{Results And Discussion}

Three phase voltage waveform is shown in fig 3 . In which voltage has been dropped of faulty phase.

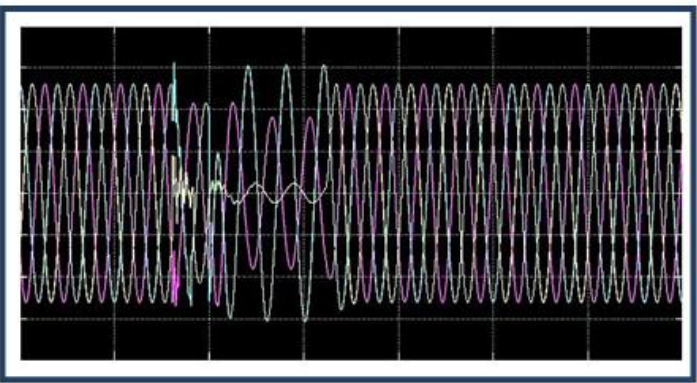

Fig. 2. 3-Phase Voltage waveform

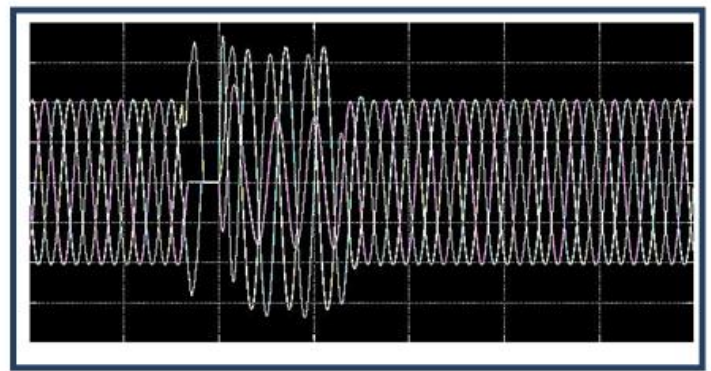

Fig. 3. 3-phase Current Waveform

Fig. 3 shows the three phase current waveform in which the value of faulty phase is rises and reached to almost 1.5 times of its original value.

The voltages and current waveforms shown in Fig. 2 and Fig. 3 extracted in 6 levels to get final approximationand details as shown in Fig.4

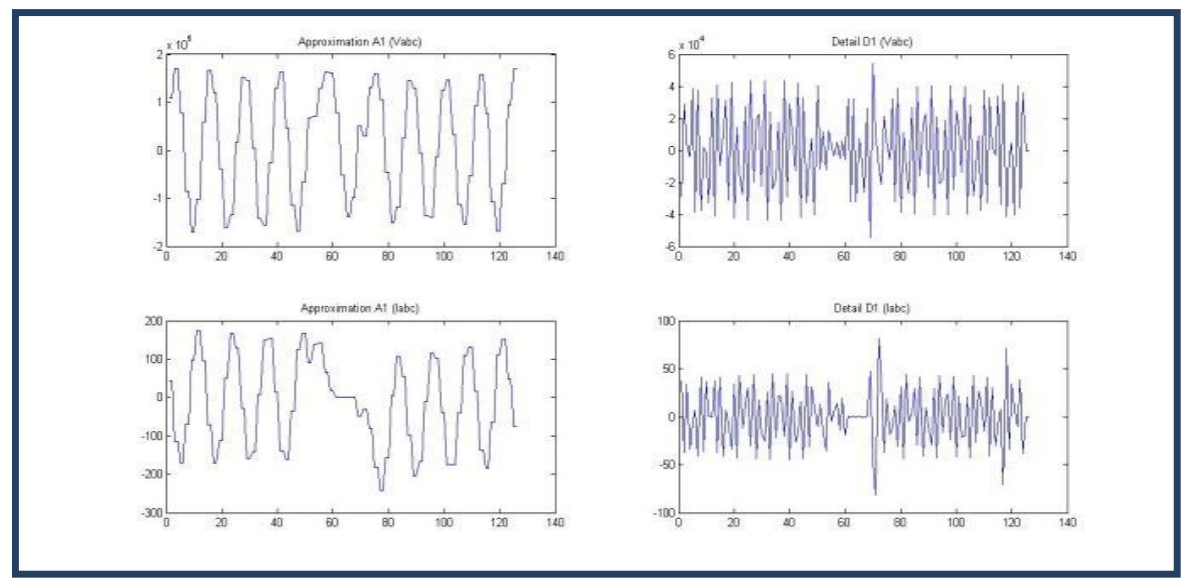

Fig. 4. Approximation (A1) and Details (D1) after 6 level decomposition with db1

Training of models is done with L-M algorithm and it gives prediction of fault location and it also classifies the fault. When this results compared with original data it is found that, for exact value of $50 \mathrm{KV}$ voltage and $375 \mathrm{KA}$ of current, the location was found is $34 \mathrm{~km}$.

\section{Conclusion}

In this paper at first, a new method to analyse the fault location for transmission network is seen. This proposed method for locating fault point offers important advantages over other methods like FFT and STFT are, due to good time and frequency localisation characteristics. Analysis results presented clearly show that particular wavelet components can be used as the features to locate the fault. Then an accurate fault location technique based on ANN is developed, as an ANN is trained to classify the fault type and separate ANNs are designed to accurately locate the actual fault position on a practical system. In this respect, three-layer feedforward ANNs and the L-M algorithm is used to adopt the weights and biases to achieve the desired non-linear mapping from inputs to outputs. Through a series of tests and modifications, it is shown that the ANNs can very accurately classify the type of fault under different system and fault conditions. The results presented herein, 
clearly show that the proposed method gives a high accuracy in fault location under a whole variety of different system and fault conditions.

Thus it can be concluded that the proposed approach based on combined WT and ANN is robust to different case studies; this is a significant advantage and can be directly attributed to the fact that WT technique effectively extracts the very crucial time-frequency features from transient signals and ANN approach is able to give a very high accuracy in the fault classification and fault location.

\section{Appendix-I}

Table 1: Transmission line parameters.

\begin{tabular}{|l|l|l|l|}
\hline i) & Conductor Size & 0.2 & ACSR \\
\hline ii) & Z1 Positive Sequence Imp & $0.16+0.4 \mathrm{j}$ & Ohm/kM \\
\hline iii) & Z0 Zero Sequence Imp & $0.394+1.25 \mathrm{j}$ & $\mathrm{Ohm} / \mathrm{kM}$ \\
\hline iv) & CTR Connected & $800 / 1$ & \\
\hline v) & PTR Connected & $132000 / 110$ & \\
\hline vi) & Earth Comp. Factor Kn & $1 / 3(\mathrm{Z} 0-\mathrm{Z} 1) / \mathrm{Z} 1$ & 0.708 \\
\hline vii) & Secondary Value Conversion Factor $(\mathrm{Cf})$ & $\mathrm{CTR} / \mathrm{PTR}$ & 0.667 \\
\hline viii) & Re/R1 & $1 / 3(\mathrm{R} 0-\mathrm{R} 1) / \mathrm{R} 1$ & 0.488 \\
\hline ix) & Xe/X1 & $1 / 3(\mathrm{X} 0 \mathrm{X} 1) / \mathrm{X} 1$ & 0.708 \\
\hline x) & Arc Resistance & & 5.000 \\
\hline xi) & Tower Footing resistance & & 15.000 \\
\hline xii) & Line parameters for protected line & $\mathrm{ZL}=4.464+11.16 \mathrm{j}$ & $12.020<68.199^{\circ}$ \\
\hline
\end{tabular}

\section{References}

[1]. Jamal Moshtagh, R. K. Aggarwal, "A New Approach To Ungrounded Fault Location In A Three-Phase Underground Distribution System Using Combined Neural Networks \& Wavelet Analysis", IEEE CCECE/CCGEI, Ottawa, May 2006

[2]. A. Abdollahi, And S. Seyedtabaii, "Transmission Line Fault Location Estimation By Fourier \& Wavelet Transforms Using ANN" The 4th International Power Engineering And Optimization Conf. (PEOCO2010), Shah Alam, Selangor, MALAYSIA: 23-24 June 2010.

[3]. Anamika Jain, "Artificial Neural Network-Based Fault Distance Locator For Double-Circuit Transmission Lines", Hindawi Publishing Corporation Advances In Artificial Intelligence, Volume 2013, Article ID 271865, 12 Pages

[4]. Springer-Verlag, "Daubechies's Ten Lectures Of Wavelets"

[5]. Zeng Xiangjun,WangYuanyuan and Xu Yao, "Faults Detection For Power Systems", IAS Annual meeting, (IEEE Industry Application Society) New Orleans, LA, pp 71-118

[6]. Seema Singh, Mamatha K R, Thejaswini S, "Intelligent Fault Identification Systems For Transmission Lines Using Artificial Neural Network", IOSR Journal Of Computer Engineering (IOSR-JCE), Volume 16, Issue 1, Ver. I (Jan. 2014), PP 23-31. 